Abanico Veterinario. Enero-Diciembre 2021; 11:1-11. http://dx.doi.org/10.21929/abavet2021.6

Artículo Original. Recibido: 10/07/2020. Aceptado: 16/01/2021. Publicado: 30/01/2021. Clave:2020-61.

\title{
Uso de melaza o aceite de soya con dos niveles de vitamina $C$ en dietas para Cavia porcellus
}

\author{
Use of molasses or soybean oil with two levels of vitamin $\mathrm{C}$ in diets for Cavia porcellus
}

\section{Salgado-Moreno Socorro*11D, Macias-Flores Mario'1D, Sánchez-Torres Laura ${ }^{31 D}$, Arredondo-Castro Mauricio ${ }^{2 I D}$, Gutiérrez-Arenas Diana ${ }^{2 I D}$, Avila-Ramos Fidel ${ }^{\star * 21 \mathrm{D}}$}

1 Universidad Autónoma de Nayarit, Unidad Académica de Medicina Veterinaria y Zootecnia. Nayarit, México. 'División Ciencias de la Vida, Universidad de Guanajuato, Programa Educativo de Medicina Veterinaria y Zootecnia. México. ${ }^{3}$ Maestría en Producción Pecuaria; Universidad de Guanajuato, Campus Irapuato-Salamanca, División Ciencias de la Vida. México. *Autor Responsable: Salgado-Moreno Socorro. ${ }^{* *}$ Autor de correspondencia: Avila-Ramos Fidel, Programa Educativo de Medicina Veterinaria y Zootecnia, Ex Hacienda El Copal km. 9; carretera Irapuato-Silao; A.P. 311; C.P. 36500; Irapuato, Guanajuato. México. coco_salgado@hotmail.com, genetica3a@gmail.com, sanchez.torres122@outlook.com, arredondo.m@ugto.mx, diana.gutierrez@ugto.mx, **ledifar@ugto.mx

\section{RESUMEN}

Los alimentos especiales para las mascotas son necesarios para aumentar su bienestar animal y mantener su buen estado de salud. El objetivo de la investigación fue conocer el efecto de la melaza o aceite de soya como fuente de energía combinada con dos niveles de vitamina $C$ en dietas para mantenimiento en cuyes. Se usaron 24 hembras distribuidas al azar en cuatro tratamientos con seis repeticiones cada uno; los animales recibieron una dieta con $3.0 \mathrm{Kcal}$ de energía digestible con aceite de soya o melaza y 100 o 300 $\mathrm{mg}$ de ácido ascórbico por $\mathrm{kg}$ de alimento. Se evaluó la ganancia de peso (GP), el consumo de alimento (CA), la permanencia capilar en anagén y la mortalidad durante 28 días. Los datos obtenidos se analizaron con un diseño completamente al azar con arreglo factorial $2 \times 2$. En los resultados no observamos interacción, pero hubo efecto en consumo de alimento al usar melaza con $100 \mathrm{mg}$ de Vitamina $\mathrm{C}$ en la primera semana, no hubo efecto de los tratamientos sobre la permanencia capilar o mortalidad. Adicionar aceite de soya o melaza como fuente concentrada de energía combinada con 100 o $300 \mathrm{mg}$ de vitamina $\mathrm{C}$ por kg de alimento no afecta el peso corporal, la mortalidad y permanencia capilar pero modifica el consumo del alimento en los cuyes.

Palabras clave: Anagén en cuyes, alimento para cuyo, pellet para cuyo, guinea pig.

\begin{abstract}
Special pet food is necessary to increase animal welfare and maintain good health. This investigation aimed to know the effect of molasses or soybean oil as an energy source with two levels of vitamin $C$ evaluated in maintenance diets. 24 females randomly distributed in four treatments with six repetitions were used, they received a diet with $3.0 \mathrm{Kcal}$ of digestible energy with soybean or molasses oil and 100 or $300 \mathrm{mg}$ of ascorbic acid per kg of food. Weight gain (WG), food consumption (FC), capillary permanence, anagen, and mortality for 28 days were evaluated. The data obtained were analysed with a completely randomized design with a $2 \times 2$ factorial arrangement. We did not observe an interaction, however, there was an effect on food consumption when using molasses with $100 \mathrm{mg}$ of Vitamin $\mathrm{C}$ in the first week. Additionally, there was no effect of the treatments on the capillary permanence or mortality. Adding soybean or molasses oil as a concentrated source of energy combined with 100 or $300 \mathrm{mg}$ of vitamin C per $\mathrm{kg}$ of food did not affect body weight, mortality, and hair permanence, but it modified food consumption in ginea pigs.
\end{abstract}

Keywords: Anagen in cuy, feedstuff for cuy, pellet for cuy, guinea pig. 


\section{INTRODUCCIÓN}

Cavia porcellus es un mamífero roedor, herbívoro de hábitos crepusculares originario de la zona andina conformada por Bolivia, Ecuador, Colombia y Perú donde forma parte de la gastronomía local (Sánchez et al., 2012). En México adopta el nombre común de Cuye - Cuyo, se reproduce para comercializarlo como animal de compañía debido a que se adapta fácilmente a espacios reducidos pero sus requerimientos alimenticios son complejos (Guevara et al., 2014). Los cuyes tienen estómago glandular degradador de carbohidratos simples por acción enzimática, pero es fermentador post gástrico debido al su ciego degradador de carbohidratos complejos a través de la fermentación bacteriana (Meza et al., 2014). Por lo tanto, su dieta debe contener cantidad adecuada de fibra, además de vitamina $C$ que su organismo no puede sintetizar debido a la falta de Lgulonolactona oxidasa (Quintana et al, 2013).

Los cuyes generalmente son alimentados con vegetales frescos como es la col, el chile pimiento, la lechuga o las zanahorias; en ocasiones se alimentan con henos, pastos, alfalfa fresca o deshidratada (Reséndiz y Hernández, 2008). En ocasiones, lo alimentan con dietas elaboradas para conejos, cerdos o con dietas mixtas (Reyes et al., 2018). Pero su alimentación deficiente puede causarles problemas hepáticos, nefríticos 0 padecimientos asociados a deficiencias nutricionales crónicas. Por ello, los alimentos balanceados para su especie cumplen con los requerimientos nutricionales específicos capaces de mejorar su vida y la condición general de su especie (León et al., 2016).

El organismo al recibir los nutrientes adecuados puede realizar los procesos metabólicos de mantenimiento eficientes, pero si tiene carencias nutricionales se pueden manifestar en diferentes formas. En cuyes las deficiencias nutricionales pueden identificarse en su pelo midiendo la etapa de crecimiento activo conocida como anagén debido a la independencia del ciclo del folículo activo y su tiempo de duración (Jackson y Ebling, 1971). Su organismo disminuye la producción de colágeno si la ingesta de ácido ascórbico baja durante dos o tres semanas debido a que sintetiza la coenzima para el precursor colágeno sub hidroxilado (Harwood et al., 1973; Grosso et al., 2013). Al recibir una dieta deficiente en vitaminas causada por ingredientes en la dieta su pelo puede modificar su crecimiento activo indicando su estado de salud.

Al balancear el alimento se mezcla proteína, energía, minerales y vitaminas de forma equilibrada (Marsanasco et al., 2011). Pero la variedad de alimentos balanceados para cuyes en México es limitada tanto en presentaciones como posiblemente en sus requerimientos nutricionales. Desarrollar un alimento de mantenimiento es adecuado para la especie, si es pelletizado se desperdicia menos, es de alta densidad, no permite la selectividad de los ingredientes por los gustos de los animales (Reséndiz y Hernández, 2008; Tarrillo et al., 2020). 
Adicionar melaza como ingrediente energético en alimentos comerciales para cuyes es común debido a su bajo costo, además, es un energético que aporta un olor dulce característico para mejorar su palatabilidad. Sin embargo, sus características pueden tener interacción con la Vitamina $\mathrm{C}$ adicionada, se dificulta su mezclado y la distribución de micro ingredientes en el producto final (Bonilla y Usca, 2015). Una alternativa como fuente energética puede ser el aceite de soya crudo que además de aportar energía a la dieta, contiene ácido linoleico y facilita la elaboración del alimento, pero a mayores costos comparados con la melaza. Por lo tanto, el objetivo de la investigación fue conocer el efecto de la melaza o el aceite de soya como fuentes energéticas combinadas con dos niveles de vitamina C $100 \mathrm{mg} / \mathrm{Kg}$ y $300 \mathrm{mg} / \mathrm{Kg}$ de alimento en dietas para mantenimiento de Cavia porcellus.

\section{Ubicación del experimento}

\section{MATERIAL Y MÉTODOS}

La investigación se realizó en la posta zootécnica de la Universidad de Guanajuato, Campus Irapuato - Salamanca, ubicada a $1,730 \mathrm{msnm}$, en clima templado con temperatura promedio de $32{ }^{\circ} \mathrm{C}$ y precipitación pluvial anual de $692 \mathrm{~mm}$. Los animales se cuidaron siguiendo las recomendaciones de la NOM-062-ZOO-1999 y el experimento se autorizó por el Comité de Investigación Agrícola-Ganadero del Laboratorio AG S.A. de C.V. (OFAG01-2019).

\section{Animales e instalaciones}

Se utilizaron 24 hembras alojados en cubículos individuales de $34 \times 54 \mathrm{~cm}$ con bebederos automáticos de niple, comederos sujetos a la base para $60 \mathrm{~g}$ de alimento, camas de aserrín de $5 \mathrm{~cm}$ de grosor y un tubo PVC de $12 \mathrm{~cm}$ de ancho por $30 \mathrm{~cm}$ de largo para evitar su estrés. Los cuyes se distribuyeron aleatoriamente en cuatro tratamientos, cada animal se identificó, se pesó y se determinó su consumo de alimento semanalmente por cuatro ocasiones. Todos los días se monitoreo el estado de salud de los animales buscando signos referentes a deficiencia de vitamina $\mathrm{C}$. Las condiciones térmicas del local se controlaron a $25 \pm 2^{\circ} \mathrm{C}$ con calentadores eléctricos (Dayton®).

\section{Elaboración de la dieta y pellet}

La dieta se elaboró a base de maíz y pasta de soya, la energía se fijó a 3.00 Mcal con melaza o aceite de soya crudo y vitamina $\mathrm{C}$ a 100 y $300 \mathrm{mg} / \mathrm{Kg}$ de alimento sugerido por la NRC de1995 (Tabla 1). Para fabricar el pellet uso una máquina peletizadora con barreno de $8.4 \mathrm{~mm}$ de entrada y $8.0 \mathrm{~mm}$ de salida, disco de $120 \mathrm{~mm}$ de longitud y $24 \mathrm{~mm}$ de grosor. El dado de la máquina era de compresión mecánica con ranura de $3.5 \mathrm{~mm}$ de ancho y $3.0 \mathrm{~mm}$ de profundidad, motor eléctrico de $3 \mathrm{Hp}$ a 127 volts y trasmisión de 100 kg por hora (Modelo: PLM0021, NS: PLED22062016). 
Tabla 1. Ingredientes de la dieta por tratamiento (\%).

\begin{tabular}{|c|c|c|c|c|}
\hline Ingredientes & T1 & T2 & T3 & T4 \\
\hline Maíz & 51.55 & 51.55 & 51.55 & 51.55 \\
\hline Alfalfa & 5.00 & 5.00 & 5.00 & 5.00 \\
\hline Pasta de soya & 21.01 & 21.01 & 21.01 & 21.01 \\
\hline Melaza & 0.00 & 3.00 & 0.00 & 3.00 \\
\hline Aceite de soya & 2.25 & 0.00 & 2.25 & 0.00 \\
\hline Pulido de arroz & 13.90 & 13.90 & 13.90 & 13.90 \\
\hline $\mathrm{CaCO}^{3}$ & 2.00 & 2.00 & 2.00 & 2.00 \\
\hline Ortofosfato & 2.59 & 2.59 & 2.57 & 2.57 \\
\hline Sal & 0.30 & 0.30 & 0.30 & 0.30 \\
\hline Premezcla vitamínica ${ }^{1}$ & 0.10 & 0.10 & 0.10 & 0.10 \\
\hline Premezcla mineral ${ }^{2}$ & 0.10 & 0.10 & 0.10 & 0.10 \\
\hline Vitamina C & 0.10 & 0.10 & 0.30 & 0.30 \\
\hline L-lisina HCL & 0.06 & 0.06 & 0.06 & 0.06 \\
\hline DL-Metionina & 0.36 & 0.36 & 0.36 & 0.36 \\
\hline Material inerte & 0.67 & 0.00 & 0.33 & 0.00 \\
\hline Ácido ascórbico & 0.01 & 0.01 & 0.03 & 0.03 \\
\hline \multicolumn{5}{|c|}{ Análisis calculado } \\
\hline Energía digestible $\left(\mathrm{Mcal} \mathrm{Kg}^{-1}\right)$ & 3.00 & 3.00 & 3.00 & 3.00 \\
\hline Proteína cruda & 17.12 & 17.12 & 17.12 & 17.12 \\
\hline Calcio & 1.28 & 1.28 & 1.28 & 1.28 \\
\hline Fósforo disponible & 0.62 & 0.62 & 0.62 & 0.62 \\
\hline Fibra & 3.00 & 3.00 & 3.00 & 3.00 \\
\hline \multicolumn{5}{|c|}{ 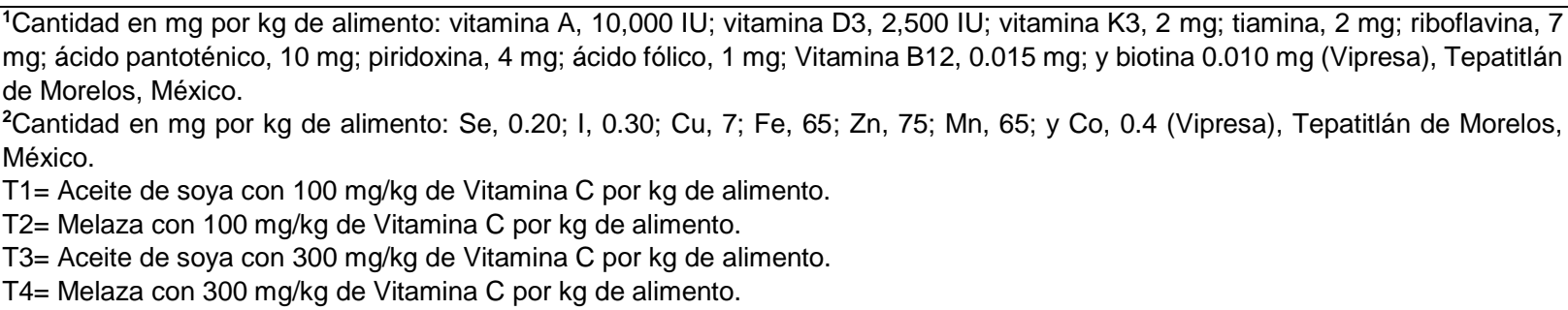 } \\
\hline
\end{tabular}

\section{Permanencia capilar}

Para conocer los cambios en el estado de salud de los cuyes se tomó una muestra de 100 pelos al iniciar y terminar el experimento. La sexta y octava vértebra dorsal se utilizaron como zonas se muestreo, un popote y una pinza hemostática se utilizaron para ejercer presión en la base del pelo y extraerlo integro. Las muestras se colocaron sobre un portaobjetos, se hidrataron, sobre ellos se colocó un cubreobjetos para conocer su estado de crecimiento en anagén (Figura 1).

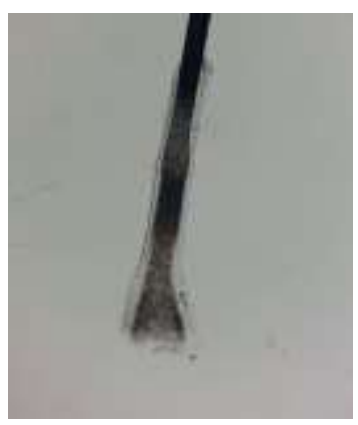

Figura 1. Base de pelo de cuye extraído de la piel en estado de anagén a 10x 


\section{Análisis estadístico}

Los datos de ganancia de peso y consumo de alimento se analizaron usando un diseño experimental completamente al azar con arreglo factorial $2 \times 2$; donde el factor A fue melaza o aceite de soya y el factor $B=100$ o $300 \mathrm{mg}$ de ácido ascórbico por kg de alimento. La permanencia capilar se evaluó en anagén comparada con la Prueba de Wilcoxon para muestras pareadas usando el procedimiento PROC UNIVARIATE. Los datos se corrieron con el programa estadístico SAS (SAS, 2010), el modelo utilizado fue:

Donde:

$$
Y_{i j k}=\mu+A_{i}+B_{j}+A B_{i j}+e_{i j k}
$$

$Y_{i j k}=$ Ganancia de peso, consumo de alimento.

$\mu=$ Media general

$A_{i}=$ Melaza o Aceite de soya

$B_{j}=$ Nivel de ácido ascórbico

$A B_{i j}=$ Interacción entre melaza, aceite de soya y nivel de ácido ascórbico.

$e_{i j k}=$ Error experimental.

\section{RESULTADOS}

No hubo efecto de la fuente concentrada de energía y nivel de vitamina $C$ sobre el peso corporal de los animales durante las cuatro semanas (Tabla 2). La adición de melaza con $100 \mathrm{mg}$ de vitamina $\mathrm{C}$ aumentó $(\mathrm{P} \leq 0.05)$ el consumo de alimento la primera semana (Tabla 3), pero en la tercera semana aumentó el consumo de alimento con aceite de soya y $100 \mathrm{mg}$ de vitamina $C(P \leq 0.05)$. En la primera y segunda semana los cuyes consumieron menos alimento $(P \leq 0.05)$, durante el experimento no hubo mortalidad y la permanencia capilar en su estado de anagén no presentó diferencias al finalizar los tratamientos (datos no mostrados).

Tabla 2. Peso corporal de cuyes (g) alimentados con dietas adicionadas con aceite de soya 0 melaza y 100 o $300 \mathrm{mg}$ de vitamina $\mathrm{C}$ por kg de alimento.

\begin{tabular}{|c|c|c|c|c|c|}
\hline \multirow{2}{*}{ Fuente de energía } & \multirow{2}{*}{$\begin{array}{c}\text { Vitamina C } \\
(\mathrm{mg} / \mathrm{kg})\end{array}$} & \multicolumn{4}{|c|}{ Semana } \\
\hline & & 1 & 2 & 3 & 4 \\
\hline \multirow{2}{*}{ Aceite de soya } & 100 & $703 \pm 152$ & $714 \pm 13$ & $737 \pm 16$ & $768 \pm 155$ \\
\hline & 300 & $603 \pm 142$ & $622 \pm 19$ & $648 \pm 118$ & $665 \pm 102$ \\
\hline \multirow{3}{*}{ Melaza } & 100 & $591 \pm 130$ & $621 \pm 123$ & $649 \pm 124$ & $697 \pm 90$ \\
\hline & 300 & $589 \pm 143$ & $611 \pm 134$ & $632 \pm 135$ & $656 \pm 143$ \\
\hline & & \multicolumn{4}{|c|}{$\operatorname{Pr}>\mathrm{F}$} \\
\hline A & & 0.2941 & 0.3558 & 0.3572 & 0.4856 \\
\hline B & & 0.3908 & 0.3664 & 0.3496 & 0.2168 \\
\hline$A^{*} B$ & & 0.4093 & 0.4661 & 0.5224 & 0.5845 \\
\hline
\end{tabular}




\section{DISCUSIÓN}

El peso corporal de los cuyes se encuentra relacionado con su raza, sexo, finalidad zootécnica y tipo de alimentación recibida. La NRC (1995) y Morales et al. (2011) evaluaron dos niveles de energía: 2.8 y $3.0 \mathrm{Mcal}$ por kg de alimento en dietas isoproteicas obteniendo mayor peso en animales al ingerir más energía. Los cuyes al no recibir una dieta adecuada para su estado fisiológico tienen menor peso, por lo tanto, al ingerir un alimento adecuado para su masa corporal aumentará como sucedió en el estudio debido al efecto de su alimentación anterior. Por lo tanto, los cuyes no aumentaron peso sólo se adaptaron a una alimentación equilibrada, su aumento de peso por día fue de $2.5 \mathrm{~g}$, ganancia adecuada para una dieta de mantenimiento comparada con animales para engorda que ganan $30 \mathrm{~g}$ de peso por día con alimentos adecuados.

Tabla 3. Consumo de alimento (g) por día de cuyes alimentados con dietas adicionadas con aceite de soya o melaza y 100 ó $300 \mathrm{mg}$ de vitamina $\mathrm{C}$ por $\mathrm{kg}$ de alimento.

\begin{tabular}{|c|c|c|c|c|c|}
\hline \multirow{2}{*}{ Fuente de energía } & \multirow{2}{*}{$\begin{array}{c}\text { Vitamina } \\
(\mathrm{mg} / \mathrm{kg})\end{array}$} & \multicolumn{4}{|c|}{ Semana } \\
\hline & & 1 & 2 & 3 & 4 \\
\hline \multirow{2}{*}{ Aceite de soya } & 100 & $25 \pm 2.4 b y$ & $30 \pm 5.4 y$ & $34 \pm 7.0 w$ & $32 \pm 3.2 w x$ \\
\hline & 300 & $23 \pm 3.2 b$ & $29 \pm 8.2$ & $33 \pm 6.5$ & $32 \pm 4.7$ \\
\hline \multirow{3}{*}{ Melaza } & 100 & $29 \pm 4.2 a$ & $30 \pm 8.2$ & $33 \pm 6.4$ & $32 \pm 2.0$ \\
\hline & 300 & $20 \pm 34 b$ & $27.7 \pm 4.7$ & $28 \pm 9.5$ & $28 \pm 6.5$ \\
\hline & \multicolumn{5}{|c|}{$\operatorname{Pr}>\mathrm{F}$} \\
\hline A & & 0.6848 & 0.7999 & 0.3348 & 0.6812 \\
\hline B & & 0.0020 & 0.5107 & 0.3731 & 0.1217 \\
\hline$A^{\star} B$ & & 0.1018 & 0.6994 & 0.6756 & 0.8999 \\
\hline
\end{tabular}

Airahuacho y Vergara (2017) reportan que los cuyes usados como mascotas deben ingerir menos de $2.9 \mathrm{Mcal}$ de ED para limitar su aumento de peso. Camino y Hidalgo (2014) evaluaron cuyes en engorda con distintos genotipos mostrando su eficiencia aumentando $15 \mathrm{~g}$ al día. En el presente estudio la dieta se balanceo para cumplir sólo el mantenimiento de los animales, por ello sólo hubo aumentos de peso mínimos, lo que se perfila como un alimento adecuado para mantenimiento en cuyes tipos B; animales con escaso desarrollo muscular, cabeza triangular, alargada, poca profundidad corporal, orejas con diferencia de tamaño y animales nerviosos (Chauca, 1997).

El consumo de alimento es una medida base para evaluar una dieta y tiene como evidencias las preferencias de los animales. Guevara et al. (2013) usaron aceite de pescado y sus cuyes comieron de 41.85 a $43.71 \mathrm{~g}$ de alimento por día. En esta investigación el consumo de alimento diario fue de 28.37 y $29.75 \mathrm{~g}$ con la melaza y aceite de soya, datos que superan a los reportados por Quintana et al. (2013) y similares a los de Guevara et al. (2013) quienes investigaron que usando plantas arbustivas en la dieta obtuvieron consumos de 24.22 a $29.50 \mathrm{~g} /$ día. Los cuyes pueden consumir hasta $56.96 \mathrm{~g}$ 
de alimento al día según lo reportado por Morales et al. (2011), pero deben usarse ingredientes adecuados para los cuyes durante la elaboración de la dieta.

La diferencia en el consumo de alimento puede variar debido a la fuente concentrada de energía usada en el experimento, su efecto extra calórico producido por el aceite de soya o la melaza pueden disminuir el consumo de alimento, incrementar el tiempo de la ingesta en el tracto digestivo mejorando su digestión. Una desventaja del aceite de soya para elaborar alimento de cuyes es su precio comparado con la melaza. Usar melaza al 30 $4 \%$ en alimentos para cuyes es común, es un aporte calórico adecuado y dosis mayores pueden ocasionar el rechazo del alimento. Además, es posible que el aroma de la melaza les pueda gustar a los cuyes.

Los cuyes necesitan vitamina $\mathrm{C}$ para cubrir sus necesidades de mantenimiento y mantener equilibrada su salud. León et al. (2016) evaluaron 450, 550 y 660 mg/kg de alimento y concluyen que $450 \mathrm{mg} / \mathrm{Kg}$ cubren los requerimientos en cuyes. Esa cantidad es una dosis alta comparada con la usada en el presente estudio de 100 y $300 \mathrm{mg} / \mathrm{kg}$ de alimento sin presentar signos negativos en los animales. Es posible que las dosis de vitamina $\mathrm{C}$ no sean las adecuadas y los animales tengan menores requerimientos. Silva y León (2015) compararon de 500 a 1100 mg de vitamina C en Kg de alimento balanceado dosis diez veces mayor a la usada y encontraron mayor consumo de alimento y ganancia de peso a los $900 \mathrm{mg}$ por $\mathrm{Kg}$ de alimento. En el presente experimento se presentó un efecto similar al incrementar de 100 a 300 mg de vitamina $\mathrm{C}$, pero solo la primera semana.

Existen diferentes aproximaciones sobre los niveles de vitamina $\mathrm{C}$ que debe contener el alimento para cuyes. La NRC (1995) sugiere $200 \mathrm{mg} / \mathrm{Kg}$ de alimento en dietas balanceadas, dosis aproximadas a las usadas en este estudio. Las dosis usada de vitamina $\mathrm{C}$ en este experimento posiblemente no causaron lesiones aparentes 0 deficiencias en los cuatro tratamientos debido al tiempo de exposición de los cuyes y manejo adecuado de los animales. Mattos et al. (2003) mencionan que los niveles de vitamina $\mathrm{C}$ deben aumentar de acuerdo con el estrés que tienen el individuo, principalmente cuando hay cambios súbitos de temperatura o manejo intenso. Guevara et al. (2014) usaron 50 y $100 \mathrm{mg} / \mathrm{Kg}$ de alimento sin tener efecto sobre el peso de los animales, resultados similares a los reportados en el presente experimento a dosis de 100 y 200 mg/Kg. Sin embargo, León et al. (2016) evaluaron 0, 450, 550 y 660 mg/kg de vitamina C con mayor consumo de alimento y peso a la dosis de $450 \mathrm{mg} / \mathrm{kg}$. La NRC (1995) sugiere $200 \mathrm{mg}$ por $\mathrm{kg}$ pero han incrementado la dosis a $800 \mathrm{mg}$ reportando aumento de peso a los 200 y $350 \mathrm{mg}$. Los resultados obtenidos en las investigaciones varían debido a los ingredientes usados para elaborar los alimentos que pueden afectar el consumo de los cuyes y sus rendimientos. 
El pelo en los cuyes tiene importancia estética debido a que indica su estado de nutrición y salud general. Vivas y Carballo (2013) recomiendan vitamina C administrada a través del alimento todos los días con dosis de acuerdo con su etapa productiva para evitar la caída de su pelo. En el presente estudio no se observaron deficiencias nutricionales en permanencia capilar en sus diferentes estados de crecimiento al final del experimento. Esto puede ser explicado por una correcta nutrición y mantención del organismo que alimenta el ciclo del colágeno para mantener el folículo piloso adecuado que proyecta una expresión fenotípica y genotípica capilar adecuada a la especie estudiada en anagén (Grosso et al., 2013).

\section{CONCLUSIÓN}

La adición de aceite de soya o melaza como fuente concentrada de energía combinada con 100 o $300 \mathrm{mg}$ de vitamina C por kg de alimento en pellet no afecta el peso corporal, consumo de alimento o permanencia capilar en cuyes. Sin embargo, es necesario continuar investigando la cantidad y clase de energía usada en alimentos especializados para la especie con distintas dosis de vitamina C.

\section{AGRADECIMIENTOS}

A todas las personas, profesores y estudiantes de la Universidad de Guanajuato por las facilidades para instalar el experimento, elaborar los alimentos y el pellet.

\section{LITERATURA CITADA}

AIRAHUACHO BFE, Vergara RV. 2017. Evaluación de dos niveles de energía digestible en base a los estándares nutricionales del NRC (1995) en dietas de crecimiento para cuyes (Cavia porcellus L). Revista de Investigaciones Veterinarias del Perú. 28(2):25526. https://doi.org/10.15381/rivep.v28i2.13079

BONILLA QS, Usca MJ. 2015. Utilización de diferentes niveles de maíz de desecho con tusa molida más melaza en la alimentación de cuyes. Revista ciencia UNEMI. 8(15):96101. ISSN: $1390-4272$. https://dialnet.unirioja.es/servlet/articulo?codigo=5269484

CAMINO JM, Hidalgo VL. 2014. Evaluación de dos genotipos de cuyes (Cavia porcellus) alimentados con concentrado y exclusión de forraje verde. Revista de investigaciones

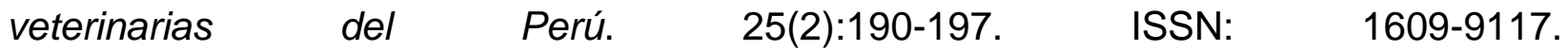
https://www.redalyc.org/pdf/3718/371834046006.pdf

CHAUCA ZL. 1997. Producción de cuyes (Cavia porcellus). La Molina, Perú. Food \& Agriculture Organización. Pp. $150 . \quad$ ISBN 92-5-304033-5. http://www.fao.org/3/W6562S/W6562S00.htm 
GUEVARA J, Díaz P, Bravo N, Vera M, Crisóstomo O, Barbachán H, Huamán D. 2013. Uso de harina de pajuro (Erithrina edulis) como suplemento en la alimentación de cuyes - Lima. Revista Peruana Química e Ingenieria Química. 16(2):21-28. https://es.scribd.com/document/396423424/Uso-de-Harina-de-Pajuro-Erythrina-EdulisComo-Suplemento-en-La-Alimentacion-de-Cuyes-Lima

GUEVARA VJ, Lozano HV, Valenzuela J. 2014. Evaluación de dos niveles de vitamina c en la alimentación de cuyes (Cavia porcellus) en crecimiento sin forraje verde. Anales Científicos. 75(2):471-474. ISSN: 2519-7398. http://dx.doi.org/10.21704/ac.v75i2.988

GROSSO G, Bei R, Mistretta A, Marventano S, Calabrese G, Masuelli L, Gazzolo D. 2013. Effects of vitamin C on health E; a review of evidence. Front biosci. 1(8):1017-1029. https://doi.org/10.2741/4160

HARWOOD R, Grant ME, Jackson DS. 1973. Collagen biosynthesis in healing wounds of guinea pigs recovering from Vitamin C deficiency. Biochemical Society Transactions. 1(5):1217-1219. https://doi.org/10.1042/bst0011217

JACKSON D, Ebling FJ. 1971. The guinea-pig hair follicle as an object for experimental observation. Journal Society Cosmetic Chemicals. 22: 701-709. http://citeseerx.ist.psu.edu/viewdoc/download?doi=10.1.1.508.1874\&rep=rep1\&type=pdf

LEÓN Z, Silva E, Wilson A, Callacna M. 2016. Vitamina C protegida en concentrado de Cavia porcellus "cuy" en etapa de crecimiento-engorde, con exclusión de forraje. Scientia Agropecuaria. 7:259-263. https://doi.org/10.17268/sci.agropecu.2016.03.14

MARSANASCO M, Márquez A, Wagner J, Alonso S, Chiaramoni N. 2011. Liposomes as vehicle for vitamins $\mathrm{E}$ and $\mathrm{C}$ : An alternative to fortify orange juice and offer $\mathrm{C}$ protection after heat treatment. Food research international. 44(9):3039-3046. https://doi.org/10.1016/j.foodres.2011.07.025

MATTOS CJ, Chauca FL, San Martín HF, Carcelén CF, Arbaiza FT. 2003. Uso del ensilado biológico del pescado en la alimentación de cuyes mejorados. Revista de investigaciones veterinarias del Perú. http://www.scielo.org.pe/scielo.php?script=sci_arttext\&pid=S1609$91172003000200001 \&$ lng $=$ es\&nrm=iso

MEZA BGA, Cabrera VPR, Morán MJJ, Meza BFFF, Cabrera VCA, Meza BCJ, Meza BJS, Cabanilla CMG, López MFX, Pincay JJL, Bohórquez BT, Ortiz DJ. 2014. Mejora de engorde de cuyes (Cavia porcellus) a base de gramíneas y forrajeras arbustivas tropicales en la zona de Quevedo, Ecuador. Idesia (Arica). 32(3):75-80. https://doi.org/10.4067/S0718-34292014000300010 
MORALES MA, Carcelén CF, Ara GM, Arbaiza FT, Chauca FL. 2011. Evaluación de dos niveles de energía en el comportamiento productivo de cuyes (Cavia porcellus) de la raza Perú. Revista de investigaciones veterinarias del Perú. 22(3):177-182. http://www.scielo.org.pe/scielo.php?script=sci_arttext\&pid=S1609-

$91172011000300001 \& \operatorname{lng}=$ es\&nrm=iso

NRC (National Research Council). 1995. Requerimientos nutritivos del cuy. 4ta ed. Washington D.C.: National Academy Press. NRC. Pp. 192. http://www.fao.org/livestock/agap/frg/nrcnut.htm

NORMA Oficial Méxicana NOM-062-ZOO-1999. Especificaciones técnicas para la producción, cuidado y uso de los animales de laboratorio. https://www.gob.mx/cms/uploads/attachment/file/203498/NOM-062-ZOO1999_220801.pdf

QUINTANA E, Jiménez R, Carcelén F, San Martín H, Ara M. 2013. Efecto de dietas de alfalfa verde, harina de cebada y bloque mineral sobre la eficiencia productiva de cuyes. Revista de Investigaciones Veterinarias del Perú. 24(4): 425-432. http://www.scielo.org.pe/pdf/rivep/v24n4/a03v24n4.pdf

RESÉNDIZ MRI, Hernández JDM. 2008. Los cuyes (Cavia porcellus) como animales de compañía. Revista Asociación de Médicos Veterinarios Especialistas en Pequeñas Especies. 19(1):22-25. https://www.imbiomed.com.mx/articulo.php?id=49879

REYES SN, Vivas J, Aguilar J, Hernández J, Caldera N. 2018. Suplementación de cobayos (Cavia porcellus L.) con forrajes frescos de morena (Morus alba) y moringa (Moringa oleífera). La calera. 18(30):7-13.

https://www.researchgate.net/publication/334066742_Suplementacion_de_cobayos_Ca via_porcellus_L_con_follajes_fresco_de_morera_Morus_alba_y_moringa_Moringa_oleif era

SAS Institute. 2010. Statistical Analysis Software SAS/STAT®. version 9.0.2, Cary, N.C., USA: SAS Institute Inc., ISBN: 978-1-60764-599-3. http://www.sas.com/en_us/software/analytics/stat.html\#

SÁNCHEZ A, Zambrano D, Torres E, Meza G. 2012. Forrajes tropicales y banano maduro (Musa paradisiaca) en el engorde de cuyes (Cavia porcellus L.) en el cantón Quevedo. Actas Iberoamericanas de Conservación Animal. 2:287-290. http://www.uco.es/conbiand/aica/templatemo_110_lin_photo/articulos/2012/Trabajo057_ AICA2012.pdf 
SILVA SJE, León GZ. 2015. Efecto de tres niveles de vitamina C de un concentrado comercial sobre el incremento de peso de Cavia porcellus "CUY" en la etapa de crecimiento y engorde. Tesis Ingeniero Agrónomo Zootecnista. Facultad de Ciencias Agropecuarias, Universidad Nacional de Trujillo, Perú. Pp. 56. http://dspace.unitru.edu.pe/bitstream/handle/UNITRU/3029/SILVA\%20SÁNCHEZ\%2C\% 20José\%20Enrique. pdf? sequence=1\&isAllowed=y

TARRILLO EBP, Mirez PKF, Bernal MW. 2018. Uso de alimento peletizado en crecimiento-engorde de cuyes mejorados (cavia porcellus) en chota. Revista Ciencia No@ndina. 1(2): 94-103. https://doi.org/10.37518/2663-6360x2020v1n2p94

VIVAS TJA, Carballo D. 2013. Especies Alternativas: Manual de crianza de cobayos (Cavia porcellus). Universidad Nacional Agraria. Pp. 49. ISBN: 9789992410226. https://www.researchgate.net/publication/311349387_Manual_de_crianza_de_cobayos 